\title{
Molecular pathogenesis of pulmonary arterial hypertension
}

\author{
Marlene Rabinovitch
}

Department of Pediatrics, Stanford University School of Medicine, Stanford, California, USA.

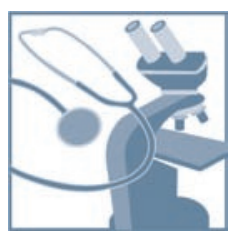

\begin{abstract}
Recent investigations have suggested that it might be possible to reverse the pathology of pulmonary arterial hypertension (PAH), a disorder that can be rapidly progressive and fatal despite current treatments including i.v. prostacyclin. This review will address the cellular and molecular processes implicated in clinical, genetic, and experimental studies as underlying the pulmonary vascular abnormalities associated with PAH. Emerging treatments are aimed at inducing apoptosis of abnormal vascular cells that obstruct blood flow and at promoting regeneration of "lost" distal vasculature.
\end{abstract}

\section{Pulmonary arterial hypertension: the disease}

Pulmonary hypertension (PH) is diagnosed quite simply by observing an elevation in mean pulmonary arterial (PA) pressure above $25 \mathrm{mmHg}$ at rest or $30 \mathrm{mmHg}$ with exercise. Patients usually present with much higher levels of PA pressure but only vague and insidious symptoms of increasing fatigue and dyspnea. Some patients are diagnosed only after syncopal episodes, which can reflect suprasystemic levels of PA pressure and low cardiac output. The causes of PH were reclassified in 2003 (as shown below) according to the clinical diagnosis (1), and some minor adjustments are being made following the 4th World Symposium on Pulmonary Hypertension in 2008.

Category I PH, also known as PA bypertension (PAH), includes idiopathic PAH (IPAH), familial PAH (FPAH), and acquired PAH (APAH), the latter of which arises in association with collagen vascular disease, PA shunts, portal hypertension, HIV infection, drugs and other toxins, and other conditions that include thyroid disorders, hemoglobinopathies, and hereditary hemorrhagic telangiectasia. This category can have significant venous or capillary involvement as a consequence of pulmonary veno-occlusive disease and pulmonary capillary hemangiomatosis, as well as persistent $\mathrm{PH}$ of the newborn. Analysis of data from a comprehensive registry in France has determined the prevalence of PAH to be 15 cases per million (2), with an average age at the time of diagnosis of $50 \pm 15$ years, a female predisposition of 2:1, an average mean PA pressure of $55 \mathrm{mmHg}$, and a mean pulmonary vascular resistance index (PVRI) of $>20$ Woods units related to a cardiac output of $2.5 \mathrm{l} / \mathrm{min}$.

Category II PH is left-sided heart disease resulting from valvular disorders or myocardial dysfunction. Category III is associated with lung disease or hypoxemia. Category IV is associated with chronic thrombotic or embolic disease. Finally, category V includes a grouping of miscellaneous disorders that are more rarely associated with $\mathrm{PH}$, including mediastinitis and sarcoid disease.

This review will focus on category I, PAH. In young children and in the neonate, $\mathrm{PAH}$ is associated with failure of the neonatal

Nonstandard abbreviations used: APAH, acquired PAH; BMP, bone morphogenetic protein; BMPRII, BMP receptor type II; DCA, dichloroacetate; EPC, endothelial progenitor cell; FPAH, familial PAH; IPAH, idiopathic PAH; Kv, voltage-gated $\mathrm{K}^{+}$; Mts1, metastasin 1; PA, pulmonary arterial/pulmonary artery; PAH, PA hypertension; $\mathrm{PH}$, pulmonary hypertension.

Conflict of interest: The author has declared that no conflict of interest exists. Citation for this article: J. Clin. Invest. 118:2372-2379 (2008). doi:10.1172/JCI33452. pulmonary vasculature to dilate at birth, in addition to abnormal muscularization of distal PAs and a striking reduction in arterial number. In older children and adults, in addition to loss of distal vessels and enhanced distal arterial muscularization, there is progressive intimal hyperplasia leading to occlusive changes in the pulmonary arteries and plexiform lesions, described below and illustrated in Figure 1. Endothelial alterations are observed in tissues from patients with $\mathrm{PH}$ and are manifest as defective von Willebrand factor, a blood glycoprotein involved in coagulation and fibrinolysis. Muscularization of distal alveolar duct and wall pulmonary arteries is associated with differentiation of pericytes into SMCs that subsequently proliferate (3). The progressive thickening of the wall of more proximal intra-acinar and pre-acinar muscular arteries and the obliteration associated with neointimal formation has been attributed to increased proliferation and migration of cells considered to be SMCs because they are $\alpha$-SMA-positive cells (4). These cells may represent a specialized subpopulation of SMCs, they may have originated as stem cells or fibrocytes (5), or they may even have arisen from ECs (6). The loss of distal vessels could be due to alterations in ECs and/or pericytes resulting in apoptosis (7). Endothelial alterations have been noted in pulmonary arteries of PAH patients (8) and in the experimental setting (9) to precede the development of muscularization of PAs. Moreover, in cell culture studies, it has been shown that ECs release factors such as FGF2, which stimulate the proliferation of SMCs (10). More recently, in PA ECs from patients with IPAH, an increase in Tie2 receptor expression and activation has been described that is related to release of serotonin from ECs. The consequence of this event can be serotonin-mediated SMC proliferation (11).

Later in disease, in association with the development of the plexiform lesion, there is also EC proliferation, leading to the formation of aberrant channels in the otherwise obliterated lumen of the vessel and in the adventitia. These channels are thought to reflect clonal expansion of apoptosis-resistant ECs (12), or they may be derived from circulating endothelial progenitor cells (EPCs) that accumulate at sites of endothelial denudation or injury and expand locally (13). PA ECs from patients with IPAH produce decreased amounts of NO. NO, synthesized largely by eNOS in ECs of the pulmonary circulation, is a vasodilator and suppressor of SMC proliferation. The reduction in NO is thought to be related to high arginase levels (14) because L-arginine, the substrate of NO synthase, is required to produce NO. The ECs from patients 


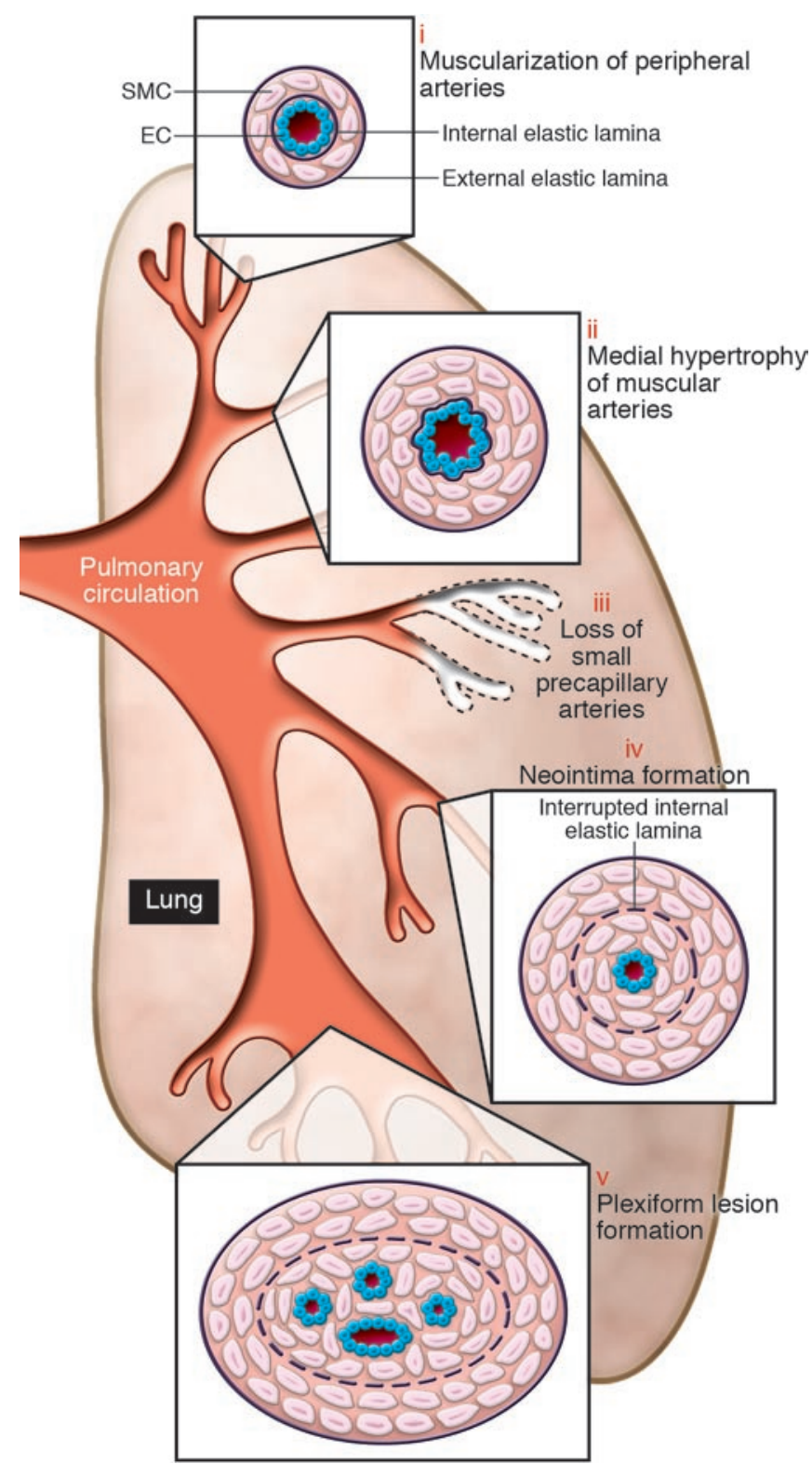

Figure 1

Pathobiology of $\mathrm{PH}$. Schema illustrating the different vascular abnormalities compared with normal pulmonary circulation, associated with $\mathrm{PH}$. This schema depicts the abnormalities throughout the pulmonary circulation, including (i) abnormal muscularization of distal precapillary arteries, (ii) medial hypertrophy (thickening) of large pulmonary muscular arteries, (iii) loss of precapillary arteries, (iv) neointimal formation that is particularly occlusive in vessels $100-500 \mu \mathrm{M}$, and (v) formation of plexiform lesions in these vessels.

with PAH are highly proliferative in response to growth factors (13) and exhibit high rates of glycolysis (15), the significance of which is poorly understood. The increased proliferation described is, however, accompanied by poor formation of endothelial tubes in culture, in keeping with the fact that these cells fail to restore the precapillary vessels that have been occluded or lost.

In addition to the pathological changes discussed above, additional features of PAH, including IPAH, consist of thicken- ing of the pulmonary adventitia and venous hypertrophy (16). Immunohistochemical studies have revealed increased expression of TGF- $\beta$, matrix proteins (such as collagen, elastin, fibronectin, tenascin-C, and glycosaminoglycans) (17), macrophages, and T cells (18) as well as inflammatory mediators such as S100A4 (also known as metastasin 1 [Mts1]) (19) and fractalkine (20).

\section{An imbalance between vasodilators and vasoconstrictors and PAH}

Patients with PAH have reduced circulating levels of the vasodilator and anti-SMC proliferative agent prostacyclin relative to levels of the vasoconstrictor and pro-SMC proliferative compound thromboxane (21). This observation led to the institution of continuous i.v. prostacyclin as a therapy for $\mathrm{PAH}$ patients. This treatment has often reduced pulmonary vascular resistance and has appreciably improved the quality of life and the survival of PAH patients (22). However, a recent meta-analysis has questioned the survival benefit of this and other therapies for PAH $(23,24)$.

Experimental studies in rats with hypoxia-induced PH (25), coupled with clinical studies documenting an increase in expression of endothelin in the lungs of patients with PAH (26), suggest that this powerful vasoconstrictor that promotes SMC proliferation and inflammation may be an important therapeutic target. There are two endothelin receptor subtypes, $\mathrm{ET}_{\mathrm{A}}$ and $\mathrm{ET}_{\mathrm{B}}$. $\mathrm{ET}_{\mathrm{A}}$ and $\mathrm{ET}_{\mathrm{B}}$ receptors are found in SMCs of blood vessels, and both can mediate vasoconstriction, but $\mathrm{ET}_{\mathrm{B}}$ receptors on $\mathrm{ECs}$ may mediate vasodilatation and endothelin clearance particularly in microvessels. Clinical trials are currently still underway comparing the effects of dual ET receptor blockers and more selective $\mathrm{ET}_{\mathrm{A}}$ receptor blockers. Recent results from studies using the dual endothelin receptor antagonist bosentan (27), as well as studies of a more selective $\mathrm{ET}_{\mathrm{A}}$ receptor antagonist (28), show alleviation of symptoms and slowing in the progression of disease in some patients. The reduced expression of NO synthase, the enzyme that generates NO (29), suggests that treatment with phosphodiesterase $V$ inhibitors such as sildenafil to prolong the NO-mediated increase in cGMP would be effective in dilating PAs (30) as well as in therapies for IPAH and APAH associated with collagen vascular disease (31), congenital cardiac left-to-right shunts (30), persistent $\mathrm{PH}$ of the newborn (32), PAH associated with hemoglobinopathies such as sickle cell disease (33), and $\mathrm{PH}$ related to thrombotic and embolic disorders (34). While one study reported that sildenafil can improve PAH in patients refractory to i.v. prostacyclin (35), there is no evidence thus far that the orally administered agents (sildenafil or ET receptor antagonists) can improve upon long-term results obtained with i.v. prostacyclin. Direct comparison of sildenafil with a dual ET receptor antagonist showed comparable results of the two therapies (36). These therapies are used in combination with anticoagulants (37). Other therapies for PAH, such as calcium channel blockers, are used only in those patients that show a beneficial acute lowering of PA pressure and resistance.

Experimental studies have also tried other vasodilators such as adrenomedullin in an attempt to reverse $\mathrm{PH}$, with good results, at least in rodents (38). Recent attention has also focused on vasoactive intestinal peptide as an important vasodilator and inhibitor of SMC proliferation in $\mathrm{PH}$, since transgenic mice that are null for vasoactive intestinal peptide develop $\mathrm{PH}$ with remodeled distal arteries and both the hemodynamic abnormality and the pathology can be reversed with administration of vasoactive intestinal peptide (39). 


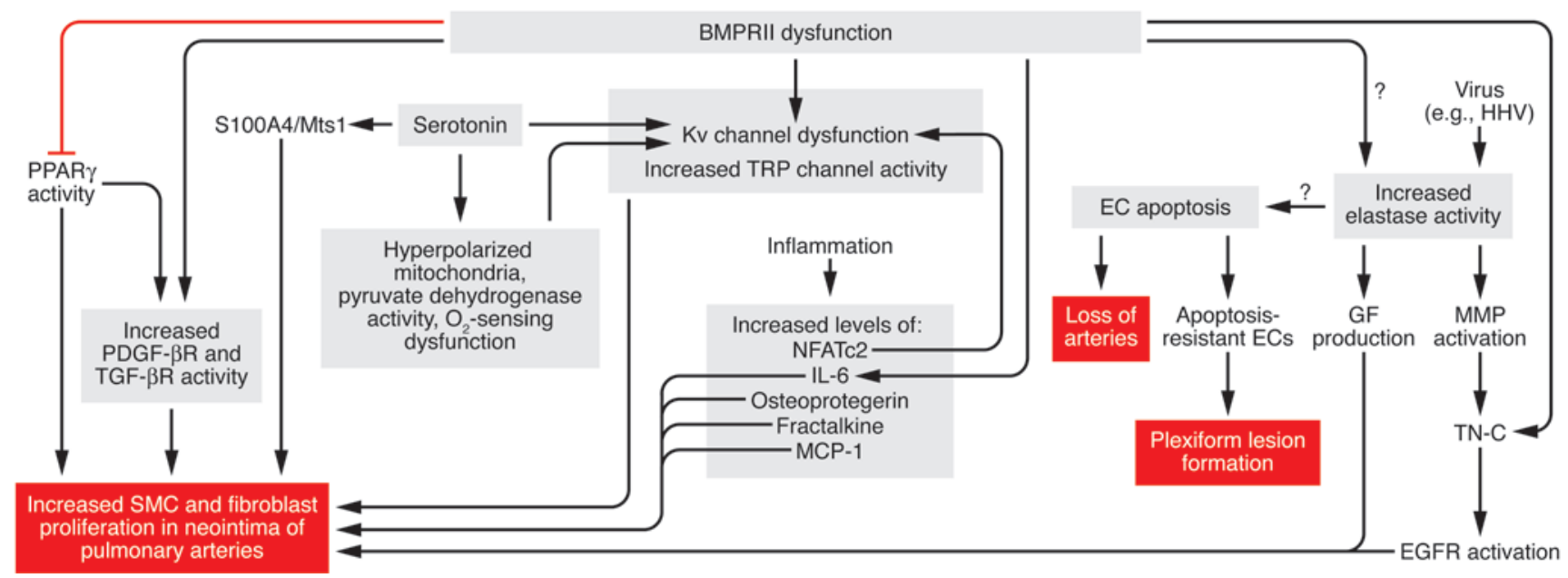

Figure 2

Schema outlining factors that converge in the molecular pathogenesis of PAH and how these may interact with BMPRII dysfunction, a known genetic defect associated with PAH. This schema focuses on factors causing increased SMC and fibroblast proliferation as well as apoptosis of ECs, causing an initial reduction in vessel number, followed by proliferation of apoptosis-resistant ECs in plexiform lesions. It shows multiple levels of interaction, with numerous factors related as described in the text. For example, serotonin stimulates both PDGF-mediated and S100A4/Mts1-mediated SMC and fibroblast proliferation and it also reduces Kv channel function, as does hyperpolarized mitochondria. BMPRII dysfunction causes Kv channel dysfunction and enhances TRP channel activity, which increases intracellular calcium levels, and may (as reflected by question mark) induce elastase activity. Viruses of the herpes family can induce elastase activity. Elastase, via activation of MMPs and tenascin $\mathrm{C}(\mathrm{TN}-\mathrm{C})$, upregulates growth factor (GF) receptors such as EGF receptors (EGFRs) and also triggers release of growth factors such as EGF from the extracellular matrix, all of which leads to SMC proliferation. BMPRII dysfunction can also increase PDGF activity, increase SMC proliferation by suppressing PPAR $\gamma$, and increase TGF- $\beta$ activity. BMPRII dysfunction can enhance inflammation via osteoprotegrin and IL-6. NFATc2 can suppress Kv channel function. Other inflammatory mediators such as fractalkine and MCP-1 can, in addition to osteoprotegrin and IL-6, increase SMC proliferation. BMPRII dysfunction can lead to EC apoptosis, as can elastase activity. EC apoptosis may predispose to the development of apoptosis-resistant ECs in plexiform lesions. MCP-1, mast cell proteinase 1; TRP, transient receptor potential Ca ${ }^{2+}$ channels.

The idea that appreciably decreasing the level of PA pressure may in and of itself reverse even severe PAH is based upon both experimental and clinical studies. For example, previous studies have shown that transplantation of a rat lung, in which monocrotaline had induced severe pulmonary vascular disease, into a normal rat was sufficient to reverse lung structural abnormalities (40). Regression of severe pulmonary vascular disease has been noted to occur in the original lung that remained in a PAH patient after single-lung transplant (41). However, there is the possibility that the regression may have been induced by immunosuppressive agents rather than by the reduction in PA pressure. In the experimental setting, immunosuppressive agents can attenuate but not reverse experimental $\mathrm{PH}$ (42). The idea of using agents that can cause major cytoskeletal changes in order to drop PA pressure has been recently revisited. Studies showing vasodilatation and reversal of $\mathrm{PH}$ by Rho kinase inhibitors such as fasudil in rodents (43) suggest that these agents should be developed for clinical use. Fasudil has indeed shown some benefit in acute testing of $\mathrm{PAH}$ patients (44). The systemic hypotensive side effects of these agents may be reduced when Rho kinase inhibitors are administered via inhalation and will need to be addressed if these agents are to be useful in treating PAH patients for a prolonged period of time.

\section{Bone morphogenetic protein receptor type II signaling and $\mathrm{PAH}$}

In concert with research leading to advances in the therapies for PAH came genetic studies showing that $60 \%$ or more of patients with FPAH $(45,46)$ and $10 \%-20 \%$ of patients with sporadic IPAH were heterozygous for a mutation in bone morphogenetic protein (BMP) receptor type II (BMPRII). BMPRII is a member of the TGF- $\beta$ superfamily of growth factor receptors. Mutations in BMPRII can be found in the ligand-binding domain, in the kinase domain, or in the long cytoplasmic tail, all of which can affect the signaling mechanism as well as interaction of the receptor with the cytoskeleton. BMPRII is expressed ubiquitously and, in association with a coreceptor, usually BMPRIA, can signal through many different pathways. The best characterized downstream signaling molecules are pSmad1/5 (47), p38 (48), pERK, JNK, and Akt/PI3K (49, 50).

In FPAH, the penetrance of BMPRII mutations is only about $20 \%$. That is, $80 \%$ of family members that carry the mutation will never develop PAH. The presence of a mutation in BMPRII is much lower $(6 \%-8 \%)$ in patients with APAH related to congenital left-toright shunts (51), and BMPRII mutations have also been observed in patients with APAH associated with toxins (appetite suppressants), but the frequency has not been established (52). Although the penetrance is low, the functional link between mutations in BMPRII and PAH is reinforced by the fact that, independent of a mutation in BMPRII, all IPAH patients have reduced BMPRII protein expression, as do, to some extent, patients with APAH (53). In addition, a reduction in the expression of the coreceptor, BMPRIA, is frequently observed in patients with IPAH (54).

The functional consequences of reduced or absent BMPRII/IA signaling in ECs and SMCs have been addressed by a number of laboratories (Figure 2). When loss of BMPRII is induced by RNA interference in PA ECs, they become susceptible to apoptosis (55). Thus it is possible that apoptosis of ECs is responsible for reduced 
peripheral alveolar duct and wall arteries, causing rarefaction of the precapillary vasculature. Loss of BMPRII causes proliferation of PA SMCs in response to TGF- $\beta 1$ and BMP2 (56), in contrast to inhibition of SMC proliferation (57) and susceptibility to apoptosis normally observed with expression of these cytokines. This observation is in keeping with the aberrant proliferative response of SMCs causing occlusive changes in intra-acinar PAs at the level of the respiratory and terminal bronchioli in PAH patients. BMP, in addition to being a negative regulator of PDGF signaling (58), is likely a negative regulator of other growth-promoting factors implicated in the pathobiology of PAH, such as EGF $(59,60)$.

In view of these studies in cultured cells, it is interesting that mice with haploinsufficiency of BMPRII (61) or with dominantnegative BMPRII (62) develop PAH associated with a relatively unimpressive degree of structural remodeling. Strategies to induce apoptosis of SMCs through statin-mediated elevation in BMP2 (63) have met with excellent experimental results in rodents, but the clinical efficacy remains to be shown. Gene therapy with BMPRII (64), however, did not appear to be sufficient to reverse monocrotaline-induced $\mathrm{PH}$.

It has also been shown that BMP4, a ligand of BMPRII/IA, induces differentiation of fetal lung fibroblasts into SMCs and inhibits their proliferation (65), suggesting that lack of BMP4 interaction with BMPRII might expand the fibroblast or myofibroblast population of cells, accounting for the adventitial and medial thickening of the PAs in PAH. It has become increasingly clear that an increase in the availability of ligands as well as a reduction in BMPRII/IA receptor signaling may lead to the development of PAH (66). For example, loss of BMP4 protects against vascular remodeling in a hypoxia model (67), which could mean that too little BMPRII or too much BMP4 in disease can have a similar effect in causing aberrant signaling. In a recent experimental study, deletion of BMPRII in cultured PA SMCs resulted in gain-of-function signaling through activin receptor IIA in conjunction with reduced BMP-2 and - 4 and increased BMP- 6 and -7 signaling (68). It would be of interest to note how, in ECs, loss of BMPRII might also result in aberrant BMP signaling. Also, while loss of BMPR1A, the coreceptor for BMPRII, was believed to contribute to the adverse pathology in IPAH patients, more recent experimental data suggest that loss of BMPRIA without concomitant reduction in BMPRII may actually protect against abnormal muscularization and loss of PAs (69).

In addition, other BMP and TGF- $\beta$ receptor family members, such as activin-like kinase type 1 (ALK1) and endoglin, that are mutated in hereditary hemorrhagic telangiectasia are also occasionally mutated in patients with PAH $(70,71)$. Additional studies focusing on aberrations in this family of receptors revealed a microsatellite instability in TGF- $\beta$ receptor II, resulting in its reduced expression and function, in patients with IPAH (72).

Thus, changes in the expression of BMPRII and BMPRIA, in the availability of ligands in concurrent abnormalities in downstream signaling events that influence gene expression, and in concurrent abnormalities in other members of the TGF- $\beta$ superfamily of receptors may all be required in the development of PAH.

In fact, it has been suggested that it is the loss of BMPRII signals that leads to an exaggeration in TGF- $\beta$ signals (73). While much attention has focused on changes in signaling patterns in response to BMPs when BMPRII is dysfunctional, few studies have specifically addressed the transcription factors and genes that are subsequently upregulated or suppressed. One candidate transcrip- tion factor is inhibitor of DNA binding 1 (ID1) (74), and another appears to be PPAR $\gamma(58,75)$.

There is evidence in cultured PA SMCs that BMP2 regulates $\operatorname{PPAR} \gamma$ transcriptional activity (58), that apoE is a putative transcription target of PPAR $\gamma$, and that mice with deletion of apoE develop PH (76). In systemic, arterial SMCs, apoE can repress proliferation by phosphorylating and internalizing the coreceptor of PDGF, namely the LDL receptor-related protein 1 (LRP1) (77). This is of further interest in light of work showing that repression of the PDGF receptor by imatinib can reverse monocrotalineinduced $\mathrm{PH}$ in rats (78) and may improve outcome in patients with end-stage PAH (79). In addition to apoE, other transcriptional targets of PPAR $\gamma$, such as adiponectin, can sequester PDGF-BB (80) and repress PA SMC proliferation. In my laboratory, we have shown that treatment of Apoe $e^{-/-}$mice with the PPAR $y$ agonist rosiglitazone reverses PAH as well as increases adiponectin levels (76). So it appears that while the antiproliferative effect of BMPRII/IA signaling in SMCs could be impeded in patients with a mutation or with impaired expression of BMPRII and/or BMPRIA, there is also the potential for rescue by activating downstream effectors such as PPAR $\gamma$. BMP2 may also be a negative regulator of transcription factors such as acute myelogenous leukemia factor 1 (AML1), which regulates serine elastase (81), an enzyme my group has implicated in the pathobiology of PAH. Other interesting targets of BMPRII implicated in proliferation of PA SMC include osteoprotegrin (82) and tenascin-C (83).

\section{Elastase activity and PAH}

My group's previous studies analyzing lung biopsy tissue from children with congenital heart defects and associated PAH suggest that elastolytic activity may be an early feature of this complication (8). Elevated serine elastase activity was subsequently documented in the monocrotaline-induced model of disease as well as in other rodent models of $\mathrm{PH}$ (84), and this led to the successful experimental use of elastase inhibitors in preventing pulmonary vascular pathology $(85,86)$ (see Experimental therapentic strategies for $\mathrm{PH}$ ). The mechanism relating elastase activity to clinical PAH is also based upon studies in cultured PA SMCs showing that heightened activity of a serine elastase leads to the release of growth factors from the extracellular matrix (10), the activation of matrix metalloproteinases, and the induction of tenascin $\mathrm{C}-\mathrm{a}$ glycoprotein associated with activation of growth factor receptors and survival pathways (59) (Figure 2). Further studies used elastase inhibitors not only to prevent but to also to reverse experimental monocrotaline-induced PH by inducing apoptosis of SMCs (87). Regression of monocrotaline-induced $\mathrm{PH}$ was subsequently also achieved by blocking a downstream effector of elastase, the EGF receptor (60). In studies using either elastase inhibitors or EGF receptor blockers, my group was able to show that there was regeneration of the distal vasculature. Similarly, treatment with a dominant-negative survivin construct (88) was highly successful in reversing PAH through SMC apoptosis.

\section{$\mathrm{K}^{+}$channel dysfunction, mitochondrial abnormalities, and PAH}

Recent studies have related aberrant signaling through BMPRII to other abnormalities that are relevant to the pathobiology of $\mathrm{PAH}$. For example, reduced expression and function of voltagegated $\mathrm{K}^{+}(\mathrm{Kv})$ channels, notably $\mathrm{Kv} 1.5$, is observed in PA SMCs from patients with IPAH as well as in APAH, and BMP2-mediated 


\section{Experimental therapeutic strategies for $\mathrm{PH}$}

\author{
Elastase inhibition \\ Kv channel openers (e.g., DCA) \\ TRP channel suppressors \\ Dominant-negative survivin \\ Statins ${ }^{\mathrm{A}}$ \\ PPAR $\gamma$ agonists \\ Growth factor (e.g., EGF, PDGF) receptor inhibition ${ }^{\mathrm{A}}$ \\ Adrenomedullin \\ Rho kinase inhibition ${ }^{\mathrm{A}}$ \\ EPCs with or without $\mathrm{eNOS}^{\mathrm{A}}$ \\ Cyclosporine \\ An increased understanding of PAH molecular pathogenesis leads \\ to new therapeutic strategies to reverse $\mathrm{PAH}$. A variety of new \\ strategies have been developed to induce apoptosis of SMCs that \\ have abnormally proliferated and to recruit ECs to regenerate distal \\ vessels. The expectation is that SMC- or EC-directed strategies will \\ have a positive impact on the other features of the disease. AThera- \\ py currently being evaluated in the clinical setting.
}

BMPRII signaling has been directly related to expression of $\mathrm{Kv}$ channels (89). Reduced expression of Kv channels favors an influx of intracellular calcium and promotes cell proliferation as well as vasoconstriction. Increased expression of Kv channels appears necessary in mediating the apoptosis associated with BMP2 signaling in SMCs, and $\mathrm{Kv}$ channel openers like dichloroacetate (DCA) as well as gene transfer of $\mathrm{Kv}$ channels have been used as experimental strategies in animal models to prevent and reverse PAH $(90,91)$ (Experimental therapentic strategies for $\mathrm{PH}$ ).

It is of interest that the fawn-hooded rat, which has a defect in serotonin metabolism and develops $\mathrm{PAH}$ in response to relative alveolar hypoxia at mile-high altitude, also has abnormal oxygen sensing in the mitochondria of SMCs, leading to reduced $\mathrm{Kv}$ channel function (92). Hyperpolarized mitochondria cause normoxic stimulation of HIF-1 $\alpha$, leading to reduced cytochrome $c$ oxidase and superoxide dismutase levels and impaired $\mathrm{Kv}$ channel expression and function (Figure 2). Reversal of the mitochondrial abnormality can be achieved through the pyruvate dehydrogenase kinase inhibitor DCA, described above as reversing $\mathrm{Kv}$ channel dysfunction (92). In addition it is reported that the serotonin can, in signaling through the 5 HT2A receptor, directly inhibit rat PA Kv channels (93).

\section{Transient receptor potential calcium channels}

Recent work has implicated transient receptor potential 3 (TRP3) and TRP6 expression in highly proliferating PA SMCs from patients with IPAH, and these studies have shown that inhibition of these channels can repress the heightened proliferation observed in these cells (94). Moreover, it was also shown that inhibition of protein kinase A or activation of cAMP might have a similar effect (95).

\section{Serotonin receptor and transporter and $\mathrm{PAH}$}

Elevated serotonin levels and serotonin transport have been implicated in the pathology of PAH, based upon studies in animals and in humans. Serotonin results in increased vasoreactivity in the fawn-hooded rat (96), and there is attenuated severity of pulmonary vascular disease in mice lacking the gene encoding the serotonin transporter (97). In contrast, overexpression of the serotonin transporter in a transgenic mouse worsens hypoxiainduced PAH (98), and when the serotonin transporter is selectively overexpressed in SMCs of transgenic mice, severe PAH ensues (99). Moreover, haploinsufficiency of BMPRII both in cultured murine PA SMCs and in transgenic mice makes them more sensitive to the pro-proliferative effects of serotonin (100). Loss of BMPRII leads to impaired repression of PDGF-mediated proliferation of SMCs (58), and this disinhibition could be further compounded by increased activity of the serotonin transporter, since this also enhances PDGF receptor $\beta$-mediated signaling (101). Other studies have shown that serotonin-mediated stimulation of the serotonin transporter and the serotonin receptors induces cyclins (102) and c-fos (103), which are critical to the PA SMC proliferative response (Figure 2).

Serotonin also stimulates the production of S100A4/Mts1, a member of the S100 family of calcium-binding proteins, by a mechanism that involves phosphorylation and nuclear translocation of the MAPK ERK1/2 and the induction of the transcription factor GATA4 (104). S100A4/Mts 1 stimulates proliferation and migration of PA SMCs (104) and is increased in neointimal lesions from patients with IPAH and PAH associated with other conditions. Moreover, a mouse that overexpresses S100A4/Mts1 spontaneously (albeit rarely) develops PAH (19).

In one study, a gain-of-function polymorphism in the serotonin transporter characterized by two long alleles was described in $65 \%$ of patients with PAH and only $27 \%$ of controls (105). This apparent modifier, however, was not observed in other studies with different populations of $\mathrm{PAH}$ patients.

\section{The proinflammatory state of the vessel wall and PAH}

Increasing attention is being focused on the proinflammatory state of the vessel wall in the progression of $\mathrm{PAH}$, but the mechanisms involved remain poorly defined. The development of PAH in a subset of patients with HIV infection may be a function of the patient's HLA class II alleles, e.g., HLA-DR6 (106). Also, a link was made between expression of human herpes virus 8 (HHV-8), associated with Kaposi sarcoma, and IPAH (107) (Figure 2). Recently my group reported that the mouse that overexpresses S100A4/Mts1 develops extensive and severe neointimal lesions following injection of the $\gamma$ murine herpes virus-68 (the murine homolog of HHV-8) (108). The HIVnef gene was also recently implicated in plexogenic pulmonary vascular lesions associated with PAH in HIV-infected patients and SIV-infected nonhuman primates (109).

Advanced occlusive lesions similar to those seen in $\mathrm{PAH}$ patients have also been produced experimentally by an immune/inflammatory $\mathrm{T}$ cell-dependent mechanism following injection of soluble antigens (110). Autoantibodies against B23, a cleavage product of a nuclear protein produced by the $\mathrm{T}$ cell enzyme granzyme $\mathrm{B}$, can distinguish the subset of patients with scleroderma who also have PAH from patients with scleroderma without PAH (111). The mechanistic significance of this biomarker is not known.

In the experimental setting, haploinsufficiency of BMPRII is associated with an increase in PAH in response to an inflammatory stimulus (112). Other experimental models of chronic inflammation, such as repeated injections of endotoxin (113) or TNF- $\alpha$ (114), result in the development of pulmonary vascular changes. A recent study has shown that in the model of PAH in which loss of arteries is induced by the combination of hypoxia and SUGEN, an 
inhibitor of VEGF (the principal growth factor for ECs), depletion of $\mathrm{T}$ cell subsets actually worsens the pathology (115). This adverse response has been attributed to unbalanced $B$ cell activity.

Heightened circulating levels of cytokines and their receptors have been demonstrated in IPAH patients, including fractalkine (20) and its cognate receptor, both of which are associated with heightened SMC proliferation (116). Other chemokines and cytokines such as stromal derived factor 1 (SDF-1) and monocyte chemoattractant protein 1 (MCP-1) that are implicated in $\mathrm{PAH}$ have been found circulating in sera from PAH patients. An interesting recent paper showed that loss of function of BMPRII leads to upregulation of the proinflammatory cytokine IL-6 (117).

Mononuclear fibrocytes have been identified as key contributors to the remodeling of the pulmonary vasculature. It is believed that these cells, which have characteristics of both fibroblasts and leukocytes, migrate into the vessel wall through the angiomata located in the expanding adventitia (5).

Most intriguing are recent studies suggesting that heightened expression of the transcription factor NFATc2 (118), which is associated with inflammatory cells, may underlie PH. Increased nuclear NFATc2 is observed in T cells from IPAH patients and in pulmonary vascular lesions, and this can lead to repression of Kv1.5 channel expression. NFATc2 can be inhibited by VIVT, a specific peptide inhibitor of calcineurin-mediated NFAT activation, and its nuclear translocation can be suppressed by cyclosporine. These agents can also attenuate monocrotaline-induced PAH.

\section{Stem cells in the pathobiology and treatment of PAH}

A major effort is being directed at understanding the mechanisms underlying pulmonary vascular regeneration following injury. In the mouse model of PAH induced by monocrotaline but not hypoxia, the recruitment of circulating stem cells appears to be protective (119). Mesenchymal stem cells can also be engineered to prevent the development of experimental $\mathrm{PH}$ in rodents, as described in studies using the endogenous vasodilator calcitonin gene-related peptide (120). Mesenchymal cells have also been delivered intratracheally to attenuate monocrotaline-induced $\mathrm{PH}$ through an obscure mechanism. In addition, these cells have been engineered to improve myocardial performance following injection into the right ventricles in rodent PAH models (121). EPCs transfected with eNOS not only prevent but also reverse PAH in rats by reestablishing connections between proximal and distal pulmonary arteries (122). This strategy of genetically engineering endogenous EPCs to express eNOS has been recently embarked upon in a clinical trial in patients with advanced PAH. Nonengineered EPCs have been used to treat clinical PAH in a pilot study showing some short-term efficacy (123). These results are at variance with studies indicating that EPCs may be the very cells that induce plexiform lesions in advanced PAH (13).

\section{The proximal pulmonary arteries and the right ventricle}

Although the focus in understanding the mechanism of PAH has been on the small pulmonary arteries $(<500 \mu \mathrm{M})$, there is evidence that changes in impedance (124), resulting from stiffening of the more proximal pulmonary arteries, may also be a critical determinant not only of the pressure but of the ability of the right ventricle to function (125). These studies also raise questions as to how BMPRII mutations associated with PAH influence the remodeling pathology of the proximal PAs and of cardiomyocytes and fibroblasts.

\section{Future directions}

Important contributions from genetics that uncover abnormalities in modifier genes of the BMPRII pathway will help elucidate the process by which IPAH develops and mechanisms regulating susceptibility in secondary forms of PAH. The role of chronic inflammation and autoimmunity will be important to pursue in novel models of PAH that recapitulate the features seen clinically. New clinical trials based upon "rescuing" altered metabolism and signaling in endothelial, smooth muscle, fibroblast, and inflammatory cells should follow from findings in experimental studies (see Experimental therapentic strategies for $\mathrm{PH}$ ).

Address correspondence to: Marlene Rabinovitch, Stanford University School of Medicine, CCSR 2245B, Stanford, California 94305-5162, USA. Phone: (650) 723-6928; Fax: (650) 723-6700; E-mail: marlener@stanford.edu.
1. Simonneau, G., et al. 2004. Clinical classification of pulmonary hypertension. J. Am. Coll. Cardiol. 43:5S-12S.

2. Humbert, M., et al. 2006. Pulmonary arterial hypertension in France: results from a national registry. Am. J. Respir. Crit. Care Med. 173:1023-1030.

3. Meyrick, B., and Reid, L. 1980. Ultrastructural findings in lung biopsy material from children with congenital heart defects. Am. J. Pathol. 101:527-537.

4. Jones, P.L., Cowan, K.N., and Rabinovitch, M. 1997. Tenascin-C, proliferation and subendothelial fibronectin in progressive pulmonary vascular disease. Am. J. Pathol. 150:1349-1360.

5. Davie, N.J., et al. 2004. Hypoxia-induced pulmonary artery adventitial remodeling and neovascularization: contribution of progenitor cells. Am.J. Physiol. Lung Cell Mol. Physiol. 286:L668-L678.

6. Frid, M.G., Kale, V.A., and Stenmark, K.R. 2002. Mature vascular endothelium can give rise to smooth muscle cells via endothelial-mesenchymal transdifferentiation: in vitro analysis. Circ. Res. 90:1189-1196.

7. Meyrick, B., Clarke, S.W., Symons, C., Woodgate, D.J., and Reid, L. 1974. Primary pulmonary hypertension: a case report including electronmicroscopic study. Br. J. Dis. Chest. 68:11-20.

8. Rabinovitch, M., et al. 1986. Pulmonary artery endothelial abnormalities in patients with congenital heart defects and pulmonary hypertension: A correlation of light with scanning electron microscopy and transmission electron microscopy. Lab. Invest. 55:632-653.

9. Rosenberg, H.C., and Rabinovitch, M. 1988. Endothelial injury and vascular reactivity in monocrotaline pulmonary hypertension. Am. J. Physiol. 255:H1484-H1491.

10. Thompson, K., and Rabinovitch, M. 1996. Exogenous leukocyte and endogenous elastases can mediate mitogenic activity in pulmonary artery smooth muscle cells by release of extracellularmatrix bound basic fibroblast growth factor. J. Cell. Physiol. 166:495-505.

11. Dewachter, L., et al. 2006. Angiopoietin/Tie2 pathway influences smooth muscle hyperplasia in idiopathic pulmonary hypertension. Am. J. Respir. Crit. Care Med. 174:1025-1033.

12. Taraseviciene-Stewart, L., et al. 2002. A bradykinin antagonist and a caspase inhibitor prevent severe pulmonary hypertension in a rat model. Can. J. Physiol. Pharmacol. 80:269-274.

13. Masri, F.A., et al. 2007. Hyperproliferative apoptosis-resistant endothelial cells in idiopathic pulmonary arterial hypertension. Am. J. Physiol. Lung Cell Mol. Physiol. 293:L548-L554.
14. Xu, W., et al. 2004. Increased arginase II and decreased NO synthesis in endothelial cells of patients with pulmonary arterial hypertension. FASEB J. 18:1746-1748.

15. Xu, W., et al. 2007. Alterations of cellular bioenergetics in pulmonary artery endothelial cells. Proc. Natl. Acad. Sci. U. S. A. 104:1342-1347.

16. Chazova, I., et al. 1995. Pulmonary artery adventitial changes and venous involvement in primary pulmonary hypertension. Am. J. Pathol. 146:389-397.

17. Jones, P., Cowan, K., and Rabinovitch, M. 1997. Progressive pulmonary vascular disease is characterized by a proliferative response related to deposition of tenascin- $\mathrm{C}$ and is preceded by subendothelial accumulation of fibronectin. Am. J. Pathol. 150:1349-1360.

18. Liptay, M.J., et al. 1993. Neointimal macrophages colocalize with extracellular matrix gene expression in human atherosclerotic pulmonary arteries. J. Clin. Invest. 91:588-594.

19. Greenway, S., et al. 2004. S100A4/Mts1 produces murine pulmonary artery changes resembling plexogenic arteriopathy and is increased in human plexogenic arteriopathy. Am. J. Pathol. 164:253-262.

20. Balabanian, K., et al. 2002. CX(3)C chemokine fractalkine in pulmonary arterial hypertension. Am.J. Respir. Crit. Care Med. 165:1419-1425. 
21. Christman, B.W., et al. 1992. An imbalance between the excretion of thromboxane and prostacyclin metabolites in pulmonary hypertension. N. Engl. J. Med. 327:70-75.

22. Barst, R.J., et al. 1996. A comparison of continuous intravenous epoprostenol (prostacyclin) with conventional therapy for primary pulmonary hypertension. The Primary Pulmonary Hypertension Study Group. N. Engl. J. Med. 334:296-301.

23. Macchia, A., et al. 2007. A meta-analysis of trials of pulmonary hypertension: a clinical condition looking for drugs and research methodology. Am. Heart J. 153:1037-1047.

24. Rich, S. 2007. The value of approved therapies for pulmonary arterial hypertension. Am. Heart J. 153:889-890.

25. Li, H., et al. 1994. Enhanced endothelin-1 and endothelin receptor gene expression in chronic hypoxia. J. Appl. Physiol. 77:1451-1459.

26. Giaid, A., et al. 1993. Expression of endothelin-1 in the lungs of patients with pulmonary hypertension. New Engl. J. Med. 328:1732-1739.

27. Sitbon, O., et al. 2003. Effects of the dual endothelin receptor antagonist bosentan in patients with pulmonary arterial hypertension: a 1-year followup study. Chest. 124:247-254.

28. Barst, R.J., et al. 2004. Sitaxsentan therapy for pulmonary arterial hypertension. Am. J. Respir. Crit. Care Med. 169:441-447.

29. Giaid, A., and Saleh, D. 1995. Reduced expression of endothelial nitric oxide synthase in the lungs of patients with pulmonary hypertension. New Engl.J. Med. 333:214-221.

30. Humpl, T., Reyes, J.T., Holtby, H., Stephens, D., and Adatia, I. 2005. Beneficial effect of oral sildenafil therapy on childhood pulmonary arterial hypertension: twelve-month clinical trial of a single-drug, open-label, pilot study. Circulation. 111:3274-3280.

31. Hayakawa, I., et al. 2005. Successful treatment with sildenafil in systemic sclerosis patients with isolated pulmonary arterial hypertension: two case reports. Rheumatol. Int. 26:270-273.

32. Juliana, A.E., and Abbad, F.C. 2005. Severe persistent pulmonary hypertension of the newborn in a setting where limited resources exclude the use of inhaled nitric oxide: successful treatment with sildenafil. Eur. J. Pediatr. 164:626-629.

33. Machado, R.F., et al. 2005. Sildenafil therapy in patients with sickle cell disease and pulmonary hypertension. Br. J. Haematol. 130:445-453.

34. Dias-Junior, C.A., et al. 2005. The effect of sildenafil on pulmonary embolism-induced oxidative stress and pulmonary hypertension. Anesth. Analg. 101:115-120

35. Kataoka, M., et al. 2005. Oral sildenafil improves primary pulmonary hypertension refractory to epoprostenol. Circ. J. 69:461-465.

36. Wilkins, M.R., et al. 2005. Sildenafil versus endothelin receptor antagonist for pulmonary hypertension (SERAPH) study. Am. J. Respir. Crit. Care Med. 171:1292-1297.

37. Thenappan, T., Shah, S.J., Rich, S., and GombergMaitland, M. 2007. A USA-based registry for pulmonary arterial hypertension:1982-2006. Eur. Respir. J. 30:1103-1110.

38. Nagaya, N., Mori, H., Murakami, S., Kangawa, K., and Kitamura, S. 2005. Adrenomedullin: angiogenesis and gene therapy. Am. J. Physiol. Regul. Integr. Comp. Physiol. 288:R1432-R1437.

39. Said, S.I., et al. 2007. Moderate pulmonary arterial hypertension in male mice lacking the vasoactive intestinal peptide gene. Circulation. 115:1260-1268.

40. O'Blenes, S.B., Fischer, S., McIntyre, B., Keshavjee, S., and Rabinovitch, M. 2001. Hemodynamic unloading leads to regression of pulmonary vascular disease in rats. J. Thorac. Cardiovasc. Surg. 121:279-289.

41. Deb, S., et al. 2006. Reversal of idiopathic pulmonary arterial hypertension and allograft pneumo nectomy after single lung transplantation. Chest. 130:214-217.

42. Nishimura, T., et al. 2001. 40-O-(2-hydroxyethyl)rapamycin attenuates pulmonary arterial hypertension and neointimal formation in rats. Am. J. Respir. Crit. Care Med. 163:498-502.

43. Oka, M., et al. 2007. Rho kinase-mediated vasoconstriction is important in severe occlusive pulmonary arterial hypertension in rats. Circ. Res. 100:923-929.

44. Ishikura, K., et al. 2006. Beneficial acute effects of rho-kinase inhibitor in patients with pulmonary arterial hypertension. Circ. J. 70:174-178.

45. Lane, K.B., et al. 2000. Heterozygous germline mutations in BMPR2, encoding a TGF-beta receptor, cause familial primary pulmonary hypertension. Nat. Genet. 26:81-84.

46. Deng, Z., et al. 2000. Familial primary pulmonary hypertension (gene PPH1) is caused by mutations in the bone morphogenetic protein receptor-II gene. Am. J. Hum. Genet. 67:737-744.

47. Rudarakanchana, N., et al. 2002. Functional analysis of bone morphogenetic protein type II receptor mutations underlying primary pulmonary hypertension. Hum. Mol. Genet. 11:1517-1525.

48. Nohe, A., et al. 2002. The mode of bone morphogenetic protein (BMP) receptor oligomerization determines different BMP-2 signaling pathways. J. Biol. Chem. 277:5330-5338.

49. Grijelmo, C., et al. 2007. Proinvasive activity of BMP-7 through SMAD4/src-independent and ERK/Rac/JNK-dependent signaling pathways in colon cancer cells. Cell Signal. 19:1722-1732.

50. Tian, Q., He, X.C., Hood, L., and Li, L. 2005. Bridging the BMP and Wnt pathways by PI3 kinase/Akt and 14-3-3zeta. Cell Cycle. 4:215-216.

51. Roberts, K.E., et al. 2004. BMPR2 mutations in pulmonary arterial hypertension with congenital heart disease. Eur. Respir. J. 24:371-374.

52. Humbert, M., et al. 2002. BMPR2 germline mutations in pulmonary hypertension associated with fenfluramine derivatives. Eur. Respir. J. 20:518-523.

53. Atkinson, C., et al. 2002. Primary pulmonary hypertension is associated with reduced pulmonary vascular expression of type II bone morphogenetic protein receptor. Circulation. 105:1627-16787.

54. Du, L., et al. 2003. Signaling molecules in nonfamilial pulmonary hypertension. N. Engl. J. Med. 348:500-509.

55. Teichert-Kuliszewska, K., et al. 2006. Bone morphogenetic protein receptor- 2 signaling promotes pulmonary arterial endothelial cell survival: implications for loss-of-function mutations in the pathogenesis of pulmonary hypertension. Circ. Res. 98:209-217.

56. Morrell, N.W., et al. 2001. Altered growth responses of pulmonary artery smooth muscle cells from patients with primary pulmonary hypertension to transforming growth factor-beta(1) and bone morphogenetic proteins. Circulation. 104:790-795.

57. Zhang, S., et al. 2003. Bone morphogenetic proteins induce apoptosis in human pulmonary vascular smooth muscle cells. Am. J. Physiol. Lung Cell Mol. Physiol. 285:L740-L754.

58. Hansmann, G., et al. 2008. An antiproliferative BMP-2/PPARgamma/apoE axis in human and murine SMCs and its role in pulmonary hypertension. J. Clin. Invest. 118:1846-1857.

59. Jones, P., Crack, J., and Rabinovitch, M. 1997. Regulation of tenascin-C, a vascular smooth muscle cell survival factor that interacts with the $\alpha v \beta 3$ integrin to promote epidermal growth factor receptor phosphorylation and growth. J. Cell Biol. 139:279-293.

60. Merklinger, S.L., Jones, P.L., Martinez, E.C., and Rabinovitch, M. 2005. Epidermal growth factor receptor blockade mediates smooth muscle cell apoptosis and improves survival in rats with pulmonary hypertension. Circulation. 112:423-431.
61. Beppu, H., et al. 2004. BMPR-II heterozygous mice have mild pulmonary hypertension and an impaired pulmonary vascular remodeling response to prolonged hypoxia. Am. J. Physiol. Lung Cell Mol. Physiol. 287:L1241-L1247.

62. West, J., et al. 2004. Pulmonary hypertension in transgenic mice expressing a dominant-negative BMPRII gene in smooth muscle. Circ. Res. 94:1109-1114.

63. Nishimura, T., et al. 2003. Simvastatin rescues rats from fatal pulmonary hypertension by inducing apoptosis of neointimal smooth muscle cells. Circulation. 108:1640-1645.

64. McMurtry, M.S., et al. 2007. Overexpression of human bone morphogenetic protein receptor 2 does not ameliorate monocrotaline pulmonary arterial hypertension. Am. J. Physiol. Lung Cell Mol. Physiol. 292:L872-L878.

65. Jeffery, T.K., Upton, P.D., Trembath, R.C., and Morrell, N.W. 2005. BMP4 inhibits proliferation and promotes myocyte differentiation of lung fibroblasts via Smad1 and JNK pathways. Am. J. Physiol. Lung Cell Mol. Physiol. 288:L370-L378.

66. Yang, X., et al. 2005. Dysfunctional Smad signaling contributes to abnormal smooth muscle cell proliferation in familial pulmonary arterial hypertension. Circ. Res. 96:1053-1063.

67. Frank, D.B., et al. 2005. Bone morphogenetic protein 4 promotes pulmonary vascular remodeling in hypoxic pulmonary hypertension. Circ. Res. 97:496-504.

68. Yu, P.B., Beppu, H., Kawai, N., Li, E., and Bloch, K.D. 2005. Bone morphogenetic protein (BMP) type II receptor deletion reveals BMP ligand-specific gain of signaling in pulmonary artery smooth muscle cells. J. Biol. Chem. 280:24443-24450.

69. El-Bizri, N., et al. 2008. Smooth muscle protein 22alpha-mediated patchy deletion of Bmpr1a impairs cardiac contractility but protects against pulmonary vascular remodeling. Circ. Res. 102:380-388

70. Harrison, R.E., et al. 2003. Molecular and functional analysis identifies ALK-1 as the predominant cause of pulmonary hypertension related to hereditary haemorrhagic telangiectasia. J. Med. Genet. 40:865-871.

71. Chaouat, A., et al. 2004. Endoglin germline mutation in a patient with hereditary haemorrhagic telangiectasia and dexfenfluramine associated pulmonary arterial hypertension. Thorax. 59:446-448.

72. Yeager, M.E., Halley, G.R., Golpon, H.A., Voelkel, N.F., and Tuder, R.M. 2001. Microsatellite instability of endothelial cell growth and apoptosis genes within plexiform lesions in primary pulmonary hypertension. Circ. Res. 88:E2-E11.

73. Morrell, N.W. 2006. Pulmonary hypertension due to BMPR2 mutation: a new paradigm for tissue remodeling? Proc. Am. Thorac. Soc. 3:680-686.

74. Wu, X., Chang, M.S., Mitsialis, S.A., and Kourembanas, S. 2006. Hypoxia regulates bone morphogenetic protein signaling through C-terminal-binding protein 1. Circ. Res. 99:240-247.

75. Crossno, J.T., Jr., et al. 2007. Rosiglitazone attenuates hypoxia-induced pulmonary arterial remodeling. Am. J. Physiol. Lung Cell Mol. Physiol. 292:L885-L897.

76. Hansmann, G., et al. 2007. Pulmonary arterial hypertension is linked to insulin resistance and reversed by peroxisome proliferator-activated receptor-gamma activation. Circulation. 115:1275-1284.

77. Takayama, Y., May, P., Anderson, R.G., and Herz, J. 2005. Low density lipoprotein receptor-related protein 1 (LRP1) controls endocytosis and c-CBLmediated ubiquitination of the platelet-derived growth factor receptor beta (PDGFR beta). J. Biol. Chem. 280:18504-18510.

78. Schermuly, R.T., et al. 2005. Reversal of experimental pulmonary hypertension by PDGF inhibition. J. Clin. Invest. 115:2811-2821. 
79. Ghofrani, H.A., Seeger, W., and Grimminger, F. 2005. Imatinib for the treatment of pulmonary arterial hypertension. N. Engl. J. Med. 353:1412-1413.

80. Arita, Y., et al. 2002. Adipocyte-derived plasma protein adiponectin acts as a platelet-derived growth factor-BB-binding protein and regulates growth factor-induced common postreceptor signal in vascular smooth muscle cell. Circulation. 105:2893-2898.

81. Wigle, D.A., et al. 1998. AML1-like transcription factor induces serine elastase activity in ovine pulmonary artery smooth muscle cells. Circ. Res. 83:252-263.

82. Lawrie, A., et al. 2008. Evidence of a role for osteoprotegerin in the pathogenesis of pulmonary arterial hypertension. Am. J. Pathol. 172:256-264.

83. Ihida-Stansbury, K., et al. 2006. Tenascin-C is induced by mutated BMP type II receptors in familial forms of pulmonary arterial hypertension. Am.J. Physiol. Lung Cell Mol. Physiol. 291:L694-L702.

84. Todorovich-Hunter, L., et al. 1992. Increased pulmonary artery elastolytic activity in adult rats with monocrotaline-induced progressive hypertensive pulmonary vascular disease compared with infant rats with nonprogressive disease. Am. Rev. Respir. Dis. 146:213-223.

85. Ye, C., and Rabinovitch, M. 1991. Inhibition of elastolysis by SC-37698 reduces development and progression of monocrotaline pulmonary hypertension. Am. J. Physiol. 261:H1255-H1267.

86. Maruyama, K., et al. 1991. Chronic hypoxic pulmonary hypertension in rats and increased elastolytic activity. Am. J. Physiol. 261:H1716-H1726.

87. Cowan, K.N., et al. 2000. Complete reversal of fatal pulmonary hypertension in rats by a serine elastase inhibitor. Nat. Med. 6:698-702.

88. McMurtry, M.S., et al. 2005. Gene therapy targeting survivin selectively induces pulmonary vascular apoptosis and reverses pulmonary arterial hypertension. J. Clin. Invest. 115:1479-1491.

89. Fantozzi, I., et al. 2006. Bone morphogenetic protein-2 upregulates expression and function of voltage-gated $\mathrm{K}+$ channels in human pulmonary artery smooth muscle cells. Am. J. Physiol. Lung Cell Mol. Physiol. 291:L993-L1004.

90. Michelakis, E.D., et al. 2002. Dichloroacetate, a metabolic modulator, prevents and reverses chronic hypoxic pulmonary hypertension in rats: role of increased expression and activity of voltage-gated potassium channels. Circulation. 105:244-250.

91. McMurtry, M.S., et al. 2004. Dichloroacetate prevents and reverses pulmonary hypertension by inducing pulmonary artery smooth muscle cell apoptosis. Circ. Res. 95:830-840.

92. Bonnet, S., et al. 2006. An abnormal mitochondrial-hypoxia inducible factor-1alpha-Kv channel pathway disrupts oxygen sensing and triggers pulmonary arterial hypertension in fawn hooded rats: similarities to human pulmonary arterial hypertension. Circulation. 113:2630-2641.

93. Cogolludo, A., et al. 2006. Serotonin inhibits voltage-gated $\mathrm{K}+$ currents in pulmonary artery smooth muscle cells: role of 5-HT2A receptors, caveolin-1, and KV1.5 channel internalization. Circ. Res. 98:931-938.

94. Yu, Y., et al. 2004. Enhanced expression of transient receptor potential channels in idiopathic pulmonary arterial hypertension. Proc. Natl. Acad. Sci. U. S. A. 101:13861-13866.

95. Zhang, S., et al. 2007. Pulmonary artery smooth muscle cells from normal subjects and IPAH patients show divergent cAMP-mediated effects on TRPC expression and capacitative Ca2+ entry. Am J. Physiol. Lung Cell Mol. Physiol. 292:L1202-L1210.

96. Le Cras, T.D., Kim, D.H., Markham, N.E., and Abman, A.S. 2000. Early abnormalities of pulmonary vascular development in the Fawn-Hooded rat raised at Denver's altitude. Am. J. Physiol. Lung Cell Mol. Physiol. 279:L283-L291.

97. Eddahibi, S., et al. 2000. Attenuated hypoxic pulmonary hypertension in mice lacking the 5-hydroxytryptamine transporter gene. J. Clin. Invest. 105:1555-1562.

98. MacLean, M.R., et al. 2004. Overexpression of the 5-hydroxytryptamine transporter gene: effect on pulmonary hemodynamics and hypoxia-induced pulmonary hypertension. Circulation. 109:2150-2155.

99. Guignabert, C., et al. 2006. Transgenic mice overexpressing the 5-hydroxytryptamine transporter gene in smooth muscle develop pulmonary hypertension. Circ. Res. 98:1323-1330.

100.Long, L., et al. 2006. Serotonin increases susceptibility to pulmonary hypertension in BMPR2-deficient mice. Circ. Res. 98:818-827.

101.Liu, Y., Li, M., Warburton, R.R., Hill, N.S., and Fanburg, B.L. 2007. The 5-HT transporter transactivates the PDGF $\{$ beta\} receptor in pulmonary artery smooth muscle cells. FASEB J. 21:2725-2734.

102.Suzuki, Y.J., et al. 2003. Activation of GATA-4 by serotonin in pulmonary artery smooth muscle cells. J. Biol. Chem. 278:17525-17531.

103.Simon, A.R., et al. 2005. 5-HT induction of c-fos gene expression requires reactive oxygen species and Rac1 and Ras GTPases. Cell Biochem. Biophys. 42:263-276.

104.Lawrie, A., et al. 2005. Interdependent serotonin transporter and receptor pathways regulate S100A4/Mts1, a gene associated with pulmonary vascular disease. Circ. Res. 97:227-235.

105.Eddahibi, S., et al. 2003. Polymorphism of the serotonin transporter gene and pulmonary hypertension in chronic obstructive pulmonary disease. Circulation. 108:1839-1844.

106. Morse, J.H., et al. 1996. Primary pulmonary hypertension in HIV infection: an outcome determined by particular HLA class II alleles. Am. J. Respir. Crit. Care Med. 153:1299-1301.

107. Cool, C.D., et al. 2003. Expression of human herpesvirus 8 in primary pulmonary hypertension. N. Engl.J. Med. 349:1113-1122.

108.Spiekerkoetter, E., et al. 2008. Reactivation of gammaHV68 induces neointimal lesions in pulmonary arteries of S100A4/Mts1-overexpressing mice in association with degradation of elastin. Am. J. Physiol. Lung Cell Mol. Physiol. 294:L276-L289.

109.Marecki, J.C., et al. 2006. HIV-1 Nef is associated with complex pulmonary vascular lesions in SHIVnef-infected macaques. Am. J. Respir. Crit. Care Med. 174:437-445.

110.Daley, E., et al. 2008. Pulmonary arterial remodeling induced by a Th2 immune response. J. Exp. Med. 205:361-372.

111.Ulanet, D.B., Flavahan, N.A., Casciola-Rosen, L., and Rosen, A. 2004. Selective cleavage of nucleolar autoantigen B23 by granzyme B in differentiated vascular smooth muscle cells: insights into the association of specific autoantibodies with distinct disease phenotypes. Arthritis Rheum. 50:233-241.

112.Song, Y., et al. 2005. Increased susceptibility to pulmonary hypertension in heterozygous BMPR2mutant mice. Circulation. 112:553-562.

113. Meyrick, B., and Brigham, K. 1986. Repeated eschrerichia coli endotocin-induced pulmonary inflammation causes chronic pulmonary hypertension in sheep. Lab. Invest. 55:164-176.

114.Stevens, T., Janssen, P.L., and Tucker, A.D. 1992. Acute and long-term TNF-alpha administration increases pulmonary vascular reactivity in isolated rat lungs. J. Appl. Physiol. 73:708-712.

115. Taraseviciene-Stewart, L., et al. 2007. Absence of $\mathrm{T}$ cells confers increased pulmonary arterial hypertension and vascular remodeling. Am. J. Respir. Crit. Care Med. 175:1280-1289.

116.Perros, F., et al. 2007. Fractalkine-induced smooth muscle cell proliferation in pulmonary hypertension. Eur. Respir. J. 29:937-943.

117. Hagen, M., et al. 2007. Interaction of interleukin6 and the BMP pathway in pulmonary smooth muscle. Am. J. Physiol. Lung Cell Mol. Physiol. 292:L1473-L1479.

118.Bonnet, S., et al. 2007. The nuclear factor of activated $T$ cells in pulmonary arterial hypertension can be therapeutically targeted. Proc. Natl. Acad. Sci. U. S. A. 104:11418-11423.

119.Baber, S.R., et al. 2006. Intratracheal mesenchymal stem cell administration attenuates monocrotalineinduced pulmonary hypertension and endothelial dysfunction. Am. J. Physiol. Heart Circ. Physiol. 292:H1120-H1128.

120.Deng, W., et al. 2006. Inhibition of vascular smooth muscle cell proliferation in vitro by genetically engineered marrow stromal cells secreting calcitonin gene-related peptide. Life Sci. 78:1830-1838.

121.Kanki-Horimoto, S., et al. 2006. Implantation of mesenchymal stem cells overexpressing endothelial nitric oxide synthase improves right ventricular impairments caused by pulmonary hypertension. Circulation. 114:I181-I185.

122.Zhao, Y.D., et al. 2005. Rescue of monocrotalineinduced pulmonary arterial hypertension using bone marrow-derived endothelial-like progenitor cells: efficacy of combined cell and eNOS gene therapy in established disease. Circ. Res. 96:442-450.

123. Wang, X.X., et al. 2007. Transplantation of autologous endothelial progenitor cells may be beneficial in patients with idiopathic pulmonary arterial hypertension: a pilot randomized controlled trial. J. Am. Coll. Cardiol. 49:1566-1571.

124. Weinberg, C.E., et al. 2004. Extraction of pulmonary vascular compliance, pulmonary vascular resistance, and right ventricular work from singlepressure and Doppler flow measurements in children with pulmonary hypertension: a new method for evaluating reactivity: in vitro and clinical studies. Circulation. 110:2609-2617.

125.Mahmud, M., and Champion, H.C. 2007. Right ventricular failure complicating heart failure: pathophysiology, significance, and management strategies. Curr. Cardiol. Rep. 9:200-208. 